\title{
D’une génération à l'autre : lecture et interprétation des paysages de bocage par les agriculteurs de trois régions laitières en Basse-Normandie (France)
}

From one generation to another: A reading and interpretation of hedgerow and field landscapes by farmers from three dairy areas in Lower Normandy (France)

De una generación a la otra : Lectura e interpretación de de los paisajes de boscaje por los agricultores de tres regiones lecheras en Baja Normandía (Francia)

\section{Maxime Marie et Philippe Madeline}

Volume 56, numéro 157, avril 2012

URI : https://id.erudit.org/iderudit/1012203ar

DOI : https://doi.org/10.7202/1012203ar

\section{Aller au sommaire du numéro}

\section{Éditeur(s)}

Département de géographie de l’Université Laval

ISSN

0007-9766 (imprimé)

1708-8968 (numérique)

Découvrir la revue

\section{Citer cet article}

Marie, M. \& Madeline, P. (2012). D’une génération à l'autre : lecture et interprétation des paysages de bocage par les agriculteurs de trois régions laitières en Basse-Normandie (France). Cahiers de géographie du Québec, 56(157), 9-28. https://doi.org/10.7202/1012203ar

\section{Résumé de l'article}

En Basse-Normandie, comme dans une grande partie des espaces ruraux européens, les transformations des systèmes de production agricole entamées au cours des années 1960 ont radicalement modifié les paysages et les rapports entretenus par les individus avec ces derniers. Cet article propose une étude des représentations paysagères des agriculteurs à partir d'une méthode d'enquête éprouvée. Nous présentons d'abord la méthode qui a conduit à l'élaboration du corpus de photographies utilisé pour analyser les représentations des exploitants agricoles. Puis nous examinons les différentes lectures des paysages qui peuvent être faites par les agriculteurs suivant un facteur générationnel. L’analyse fine des discours produits par ces derniers nous conduit ainsi à identifier des différences marquées entre les exploitants ayant participé aux principales phases d'intensification agricole durant les années 1970 et ceux, plus jeunes, installés dans les années 1980. Enfin, l'étude des discours sur les friches et les cultures révèle la place centrale des identités professionnelles dans la construction des représentations paysagères des agriculteurs.
Tous droits réservés (c Cahiers de géographie du Québec, 2012
Ce document est protégé par la loi sur le droit d'auteur. L'utilisation des services d'Érudit (y compris la reproduction) est assujettie à sa politique d'utilisation que vous pouvez consulter en ligne.

https://apropos.erudit.org/fr/usagers/politique-dutilisation/ 


\section{D'une génération à l'autre: lecture et interprétation des paysages de bocage par les agriculteurs de trois régions laitières en Basse-Normandie (France)}

\author{
From one generation to another: A reading \\ and interpretation of hedgerow and field \\ landscapes by farmers from three dairy \\ areas in Lower Normandy (France)
}

De una generación a la otra: Lectura e interpretación de de los paisajes de boscaje por los agricultores de tres regiones lecheras en Baja Normandía (Francia)

\author{
Maxime MARIE et Philippe MADELINE \\ Laboratoire ESO-CAEN, UMR ESO 6590 CNRS \\ Université de Caen Basse-Normandie \\ Maxime_Marie@unicaen.fr \\ Philippe.Madeline@unicaen.fr
}

\section{Résumé}

En Basse-Normandie, comme dans une grande partie des espaces ruraux européens, les transformations des systèmes de production agricole entamées au cours des années 1960 ont radicalement modifié les paysages et les rapports entretenus par les individus avec ces derniers. Cet article propose une étude des représentations paysagères des agriculteurs à partir d'une méthode d'enquête éprouvée. Nous présentons d'abord la méthode qui a conduit à l'élaboration du corpus de photographies utilisé pour analyser les représentations des exploitants agricoles. Puis nous examinons les différentes lectures des paysages qui peuvent être faites par les agriculteurs suivant un facteur générationnel. L’analyse fine des discours produits par ces derniers nous conduit ainsi à identifier des différences marquées entre les exploitants ayant participé aux principales phases d'intensification agricole durant les années 1970 et ceux, plus jeunes, installés dans les années 1980. Enfin, l'étude des discours sur les friches et les cultures révèle la place centrale des identités professionnelles dans la construction des représentations paysagères des agriculteurs.

\section{Mots-clés}

Paysage agricole, agriculteurs, représentation paysagère, facteur générationnel.

\section{Abstract}

Following a process initiated during the 1960s in a large number of Europe's rural areas, the transformation of agricultural production systems brought about significant changes in both the landscape of Lower Normandy and the relationship people had with it. The purpose of this paper is to apply proven survey methodology to analyze how farmers see their landscapes. We begin by explaining the method used to select the photographic corpus used as the basis for the study. We then examine the way two generations of farmers interpret their landscapes. Our discourse analysis reveals significant differences between these farmers, differences that 
reflect a major shift in perceptions between those who were part of the agricultural productivism reforms of the 1970s, and members of the younger generation who began working in agriculture in the mid-1980s. Furthermore, our analysis of their discourse on wastelands and crops highlights the crucial importance of professional identity in the ways these farmers construct their particular representations of their landscapes.

\section{Keywords}

Agricultural landscapes, farmers, representation of landscapes, generational factors.

\section{Resumen}

En Baja Normandía, como en gran parte de los espacios rurales europeos, las transformaciones de los sistemas de producción agrícola comenzadas en los años 1960 han modificado radicalmente los paisajes y las relaciones entre estos con los individuos. Este artículo propone un estudio de las representaciones paisajísticas de los agricultores, gracias a un método validado de encuestas. Se presentan, primero, el método para constituir el corpus de fotografías utilizado para el análisis de las representaciones de los productores agrícolas. Luego, se examinan las diferentes lecturas probables de paisajes presentadas por los agricultores según un factor generacional. El análisis detallado de esos discursos permite establecer diferencias marcadas entre los cultivadores que participaron en las etapas principales en la intensificación agrícola de los años 1970, con los más jóvenes instalados en los años 1980. Finalmente se presenta el resultado del estudio de los discursos sobre los eriales y los cultivos, lo que revela el espacio central de las identidades profesionales en la construcción de las representaciones paisajísticas de los agricultores.

\section{Palabras claves}

Paisaje agrícola, agricultores, representación del paisaje, factor generacional.

\section{Introduction}

Depuis la fin des années 1960, les transformations des structures agricoles et sociales des espaces ruraux de l'Ouest français ont profondément bouleversé les paysages. Le rôle des agriculteurs, fondamental dans ces mutations, est ici examiné sous l'angle de leurs représentations. Au moment où de nouvelles demandes concernant l'agriculture et les paysages émanent des sociétés, les recherches sur les rapports des agriculteurs au paysage permettent de mieux cerner la manière dont ils se le représentent et agissent concrètement sur lui (Calvo-Iglesias et al., 2006).

Dans les bocages de l'Ouest français où la SAU (surface agricole utilisée) atteint souvent plus de $80 \%$ des superficies communales, les agriculteurs peuvent être considérés comme des «producteurs» de paysage dans la mesure où ils sont les acteurs essentiels des dynamiques paysagères (Rémy, 2005). Au-delà des grands éléments structurant les représentations et les postures des agriculteurs quant au paysage (Droz et al., 2009; 
Marie, 2009), cette étude ${ }^{1}$ nous conduit à nous interroger sur l'existence d'un clivage générationnel au sein du monde agricole. En effet, nous formulons l'hypothèse que les différentes phases de modernisation agricole des années 1970 et 1980, comme les recompositions des rôles sociaux et territoriaux de l'agriculture en cours depuis le milieu des années 1990 (débat autour de la multifonctionnalité, la reterritorialisation des productions, etc.), induisent une modification profonde du contexte de socialisation dans lequel évoluent les agriculteurs et donc une transformation de leurs identités professionnelles (Lémery, 2003), mais aussi de leurs rapports à l'espace et au paysage, et à travers eux, au reste de la société (Hervieu et Viard, 2001).

La réflexion porte aussi sur le rôle que ces systèmes perceptifs et représentatifs peuvent recouvrir dans les mécanismes de décision et de mise en œuvre de l'action paysagère. À un moment où le modèle productiviste est sévèrement remis en question et où les demandes sociales vis-à-vis du paysage et du cadre de vie se font de plus en plus pressantes, les informations recueillies permettent d'éclairer les conflits opposant les agriculteurs aux autres usagers de l'espace rural, ainsi que les raisons des échecs et des réussites des politiques de préservation et de gestion des paysages bocagers dans la France de l'Ouest.

\section{Terrain et méthodologie}

Afin d'éviter les écueils de certaines recherches qualitatives sur les paysages (Luginbühl, 1995; Becker, 2007; Maresca, 2007), nous appuyons notre approche méthodologique sur des entretiens et la distribution, aux agriculteurs, d'un appareil photographique jetable. Si le recours à la photographie est ancien dans les recherches menées en anthropologie et en ethnologie (Collier et Collier, 1986; Moore, 1997), elle est moins répandue en géographie, bien qu'elle s'avère particulièrement bien adaptée aux travaux sur les représentations paysagères. Nous reproduisons ici un mode opératoire d'abord utilisé par Yves Michelin, chercheur à l’École nationale d'ingénieurs des travaux agricles (ENITA), auprès d'élus locaux de la région Auvergne et repris par d'autres chercheurs, notamment ceux de l'équipe Métafort (Michelin, 1998; Lelli et Paradis, 2005 ; Ménadier, 2010). Les résultats obtenus par ces derniers nous ont convaincus de l'intérêt de cette méthode même si les populations, l'échelle d'analyse et le traitement des résultats diffèrent. Cette technique permet d'écarter partiellement la subjectivité du chercheur en mettant l'accent sur la participation et l'autonomie des individus prenant part à l'étude. En effet, trop souvent attachés aux méthodes d'entretien qui négligent l'approche quantitative, les nombreux travaux qui concernent les représentations paysagères des individus sur des échantillons réduits ne permettent que difficilement d'identifier des tendances générales (Luginbühl, 1989; Cadiou, 1991 ; Cadiou et Luginbühl, 1995).

L'enquête présentée ici a été réalisée dans trois petites régions agricoles de BasseNormandie: le Sud Pays d'Auge, le Bocage Virois et le Sud Manche (figure 1). Dans ces trois espaces d'élevage et de bocage, les exploitations agricoles sont très majoritairement laitières.

1 Les recherches présentées ici ont été réalisées dans le cadre d'une thèse de géographie portant sur les dynamiques et les logiques d'organisation spatiale des activités agricoles en zone de bocage (Marie, 2009) (thèse financée par le ministère français de l'Enseignement supérieur et de la Recherche). 
Ces terrains ont donc été retenus en raison de leurs caractéristiques laitières et bocagères communes, mais aussi en raison de certaines dissemblances: densité d'exploitations agricoles (forte dans le Sud Manche, moyenne dans le Bocage Virois, assez faible dans le Sud Pays d'Auge), niveau d'intensification des systèmes fourragers (très élevé dans le Sud Manche, élevé dans le Bocage Virois, relativement faible dans le Sud Pays d'Auge), degré de morcellement parcellaire (élevé dans le Sud Manche, moyen dans le Bocage Virois, faible dans le Sud Pays d'Auge) (Bermond, 2004; Marie, 2009).

\section{Figure 1 Localisation des espaces d'étude}

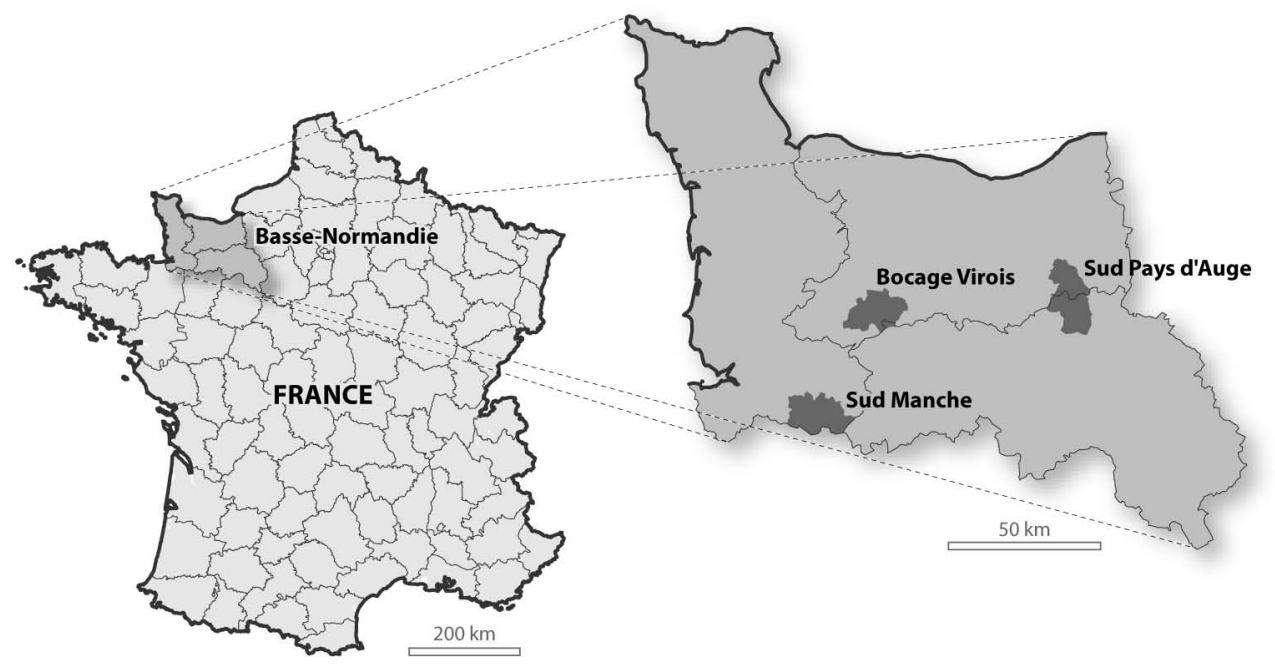

L'appareil photo jetable fourni à chaque agriculteur est accompagné d'une fiche-guide présentant l'objectif général de l'exercice et les consignes de prise de vue (Marie, 2007). Seuls les chefs d'exploitation ont été sollicités, les conjoints et les enfants des agriculteurs n’ont donc pas été associés à la mise en œuvre de l'expérience. Cette fiche-guide est composée d'une série de questions ou de consignes auxquelles doit répondre notre interlocuteur par des clichés (trois prises de vue au maximum pour chaque question) et un fond de carte de son exploitation, sur lequel chaque prise de vue doit être exactement localisée. Afin d'analyser les représentations paysagères, la fiche-guide oriente donc vers sept types de clichés selon les entrées suivantes :

- les éléments relatifs au registre affectif, c’est-à-dire les éléments des paysages participant à l'identification des individus à l'espace qu'ils habitent et façonnent;

- les éléments paysagers rejetés, sur lesquels toute amélioration sera vécue comme positive du point de vue de la qualité des paysages;

- les éléments valorisables d'un point de vue agricole et paysager dans le cadre du développement de l'agrotourisme; ces éléments renseignent sur l'identification par les agriculteurs des demandes émanant de la société;

- les éléments attribués au passé: comment les agriculteurs se représententils les évolutions passées des paysages? comment celles-ci sont-elles exprimées dans le paysage? 
- les évolutions paysagères en cours et futures: quelles sont les composantes des paysages que les agriculteurs voient comme les plus fragiles et relient au futur de l'activité?

- ce qui est identifié comme une «belle» parcelle pour les agriculteurs : quelle est la parcelle idéale vers laquelle tendent les aménagements parcellaires?

- les éléments que les agriculteurs considèrent comme constitutifs de leur outil de travail (bâtiments agricoles, parcelles, aménagement divers).

Une campagne d'entretiens menée avant la distribution des appareils photo a permis de cerner les relations des exploitants agricoles avec les paysages qui les entourent. Après la distribution de l'appareil, un second entretien a porté sur le sens qu'ils donnaient à leurs clichés, utilisés ici comme support d'entretien (Harper, 2002; Papinot, 2007 ; Marie, 2009). Cette méthode, qui permet de produire un matériau scientifique et empirique très intéressant, nécessite cependant un lourd investissement en temps et une forte implication de la part des agriculteurs. C'est la raison pour laquelle seulement 15 ont été sollicités ( 5 dans chaque zone d'étude).

Distribués au mois de mars 2004 dans le Pays d'Auge et le Sud Manche et en mai 2008 dans le Bocage Virois, les appareils photo et les fiches-guides devaient être récupérés dans les trois semaines. Malgré la durée de l'exercice - un temps moyen de cinq heures -, la plupart des clichés ont été effectués en moins de deux semaines. Cet empressement s'explique par la motivation et l'intérêt portés à la démarche. Il traduit bien, de la part des agriculteurs rencontrés, un "souci paysager » et une volonté de s'exprimer sur le paysage (De la Soudière, 1984; Bertrand et Bories, 2004; Lelli et Paradis, 2005).

Deux temps caractérisent l'interprétation des clichés. Une première phase a permis de recenser les formes paysagères consciemment photographiées : un bâtiment agricole, une carcasse de caravane, une haie... L'observation minutieuse des photographies apporte des renseignements insoupçonnés : le choix du cadrage sur des plans larges, par exemple, indique la façon dont sont inconsciemment considérées certaines formes paysagères. Ainsi, sur une vue générale d'un paysage de bocage, la présence dans les premiers plans d'une forte proportion de terres labourées peut fournir un indice de la manière dont le "photographe» a assimilé cet élément aux modèles paysagers auxquels il adhère, et donc nous renseigner sur ses représentations paysagères. Enfin, le temps que les agriculteurs ont mis pour effectuer les prises de vue et les efforts de cadrage constituent autant d'indicateurs de leur motivation et de leur sensibilité à l'objet paysager.

Après l'identification des formes paysagères les plus récurrentes des clichés, un tri par catégories a conduit à leur interprétation: haies ou bocage, prairies, cultures ou labours, vergers haute-tige, bois ou friches, bâti rural traditionnel, bâtiments agricoles modernes, machines et outils agricoles, animaux, et témoins des usages non agricoles de l'espace rural. Chaque cliché a ensuite été «décortiqué» suivant cette grille de lecture, et des corrélations ont été effectuées entre les formes paysagères représentées et le registre dans lequel elles apparaissent (affectif, rejet, évolutif, outils de travail, valorisation touristique...). 
Les entretiens ont permis d'approfondir notre compréhension des clichés et de les confronter aux consignes de prise de vue figurant dans la fiche-guide. Les clichés, qui répondent directement aux consignes, sont l'expression d'un positionnement des agriculteurs quant au paysage, aux évolutions passées et futures des formes le constituant. Durant cette seconde phase, les prises de vue doivent ainsi être considérées comme un support de discussion avec les agriculteurs leur permettant de construire un discours sur le paysage, notion qui reste parfois difficile à aborder sans recours à l'iconographie (Harper, 2002; Conord, 2007). Tous les entretiens ont été enregistrés et intégralement retranscrits, mais nous avons choisi ici de n'exploiter que de manière qualitative les matériaux récoltés. Les extraits qui ponctuent l'article n’ont donc ici qu'une fonction illustrative et démonstrative.

En résumé, le principe de cette démarche méthodologique réside donc dans l'analyse de manière systématique des clichés composant le corpus photographique. Dans cette analyse, les prises de vue sont décrites a posteriori par une grille descriptive issue de la typologie des formes paysagères récurrentes dans les deux premiers plans. Les résultats de ce premier travail sont enfin complétés de manière qualitative par les entretiens réalisés avec les agriculteurs à partir de leurs clichés sans qu'il ne soit fait référence à la première phase du travail d’analyse.

Le corpus de photographies ainsi obtenu est important : il compte en effet un peu plus de 240 clichés exploitables. L'examen des prises de vue suivant une grille de comptage des occurrences de chaque forme paysagère révèle ainsi une répartition thématique intéressante (figure 2).

Les registres les plus motivants relevés par les agriculteurs concernent l'identification, les évolutions passées des paysages et leur outil de production (respectivement 40, 40 et 43 clichés au total). Le fait que les agriculteurs aient atteint, voire dépassé, le maximum autorisé de trois prises de vue par consigne semble révéler leur forte mobilisation autour de ces sujets. En revanche, au registre des thèmes qui ne les ont pas inspirés figurent ceux relatifs aux évolutions futures et aux éléments qu'ils rejettent dans le paysage (le cas de la «belle parcelle» est un peu particulier, car un seul cliché était demandé).

\section{Figure 2 Le corpus de photographies réalisées par les agriculteurs normands}
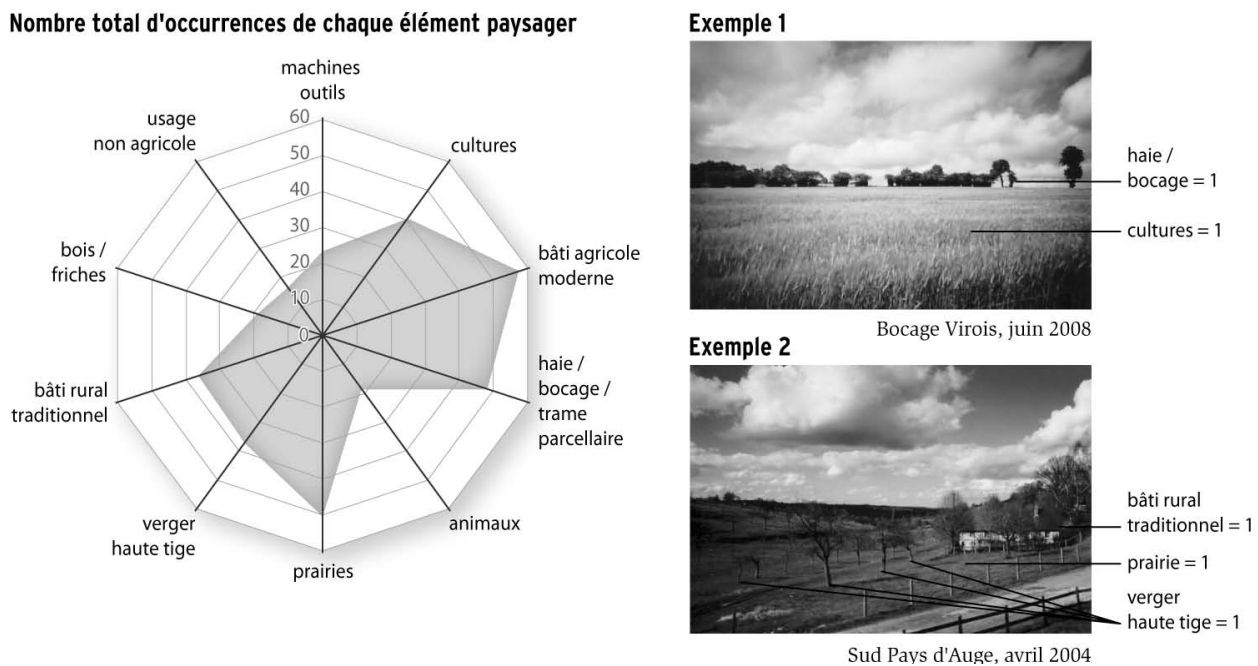
Les éléments paysagers présents sur les photographies sont assez divers : bois-friches, labours, prairie-verger haute tige, haies/bocage et bâti. Les éléments les plus représentés sont les bâtiments (27\% des clichés) puis l'association prairie-verger (25\%) et la maille bocagère $(14 \%)$. La répartition des éléments paysagers par registre met en valeur les associations entre les formes paysagères et les thèmes auxquels les agriculteurs les rattachent. On constate, par exemple, que les clichés des prairiesvergers sont particulièrement présents pour le registre de la valorisation touristique des espaces ruraux. Il en va de même pour les bâtiments agricoles ou les machines agricoles, qui apparaissent de façon très franche dans le registre de l'outil de travail.

\section{Des clichés aux représentations: la traduction d'un clivage générationnel}

De nombreux travaux insistent sur la diversité interne du monde agricole et sur l'importance de ne pas traiter le groupe social que constituent les agriculteurs comme un tout homogène au sein de la société française (Hervieu et Viard, 2002; Rémy, 2006). Dans le domaine des représentations associées aux paysages chez les agriculteurs, un certain nombre d'études montrent l'intérêt de dépasser une vision monolithique du monde agricole pour s'intéresser aux facteurs qui contribuent à expliquer les différentes postures observables au sein du groupe (Gravsholt Busck, 2002 ; Miéville-Ott et Droz, 2010).

Étant donné le nombre restreint d'agriculteurs ayant participé à l'enquête, la diversité de leur propriétés sociales et les caractéristiques de leurs exploitations (15 individus dont une seule femme), nous avons choisi de ne diviser l'échantillon qu'en deux sousgroupes (tableau 1). Pour la constitution de ces deux sous-groupes, le critère d'âge a été préféré à celui du zonage géographique qui montre en fait peu de différences significatives entre les trois espaces d'étude (Marie, 2009). L'âge moyen des individus ayant participé à l'enquête est légèrement supérieur aux moyennes constatées dans l'ensemble de la population agricole des trois zones d'étude. Cela s'explique par la présence d'un agriculteur âgé dans les échantillons de chacune des régions (un agriculteur d'environ 60 ans sur les cinq interrogés), ce qui cause une légère surreprésentation de ces derniers et affecte mécaniquement la moyenne d’âge (de 3 ans supérieure aux chiffres des statistiques officielles).

Le premier groupe se compose d'agriculteurs âgés de plus de 48 ans (âge médian de l'ensemble de l'échantillon), installés avant 1980 et dont la moyenne d'âge est d'environ 60 ans. Les exploitants du second sous-groupe ont moins de 48 ans (40 ans d'âge moyen) et se sont installés après 1984. Au regard de notre analyse, la date d'installation est un paramètre qui pèse lourdement, car elle rend compte du contexte agricole et sociétal dans lequel l'individu a évolué. En effet, être agriculteur aujourd'hui ou dans les années 1970 ne génère pas les mêmes comportements face à la conduite de l'exploitation ni la même attitude face au paysage (Chosson, 2003). Les principales actions d'aménagement parcellaire et foncier, l'introduction du maïs fourrage dans le cadre de l'intensification fourragère, ainsi que les constructions de nouveaux bâtiments agricoles ont débuté dans les années 1960 et se sont prolongées tout au long des années 1970. À cette époque, le paysage était loin d'être au centre des préoccupations des aménageurs, et ce mot n’apparaissait pas dans les textes officiels (Ambroise et al., 2000). 


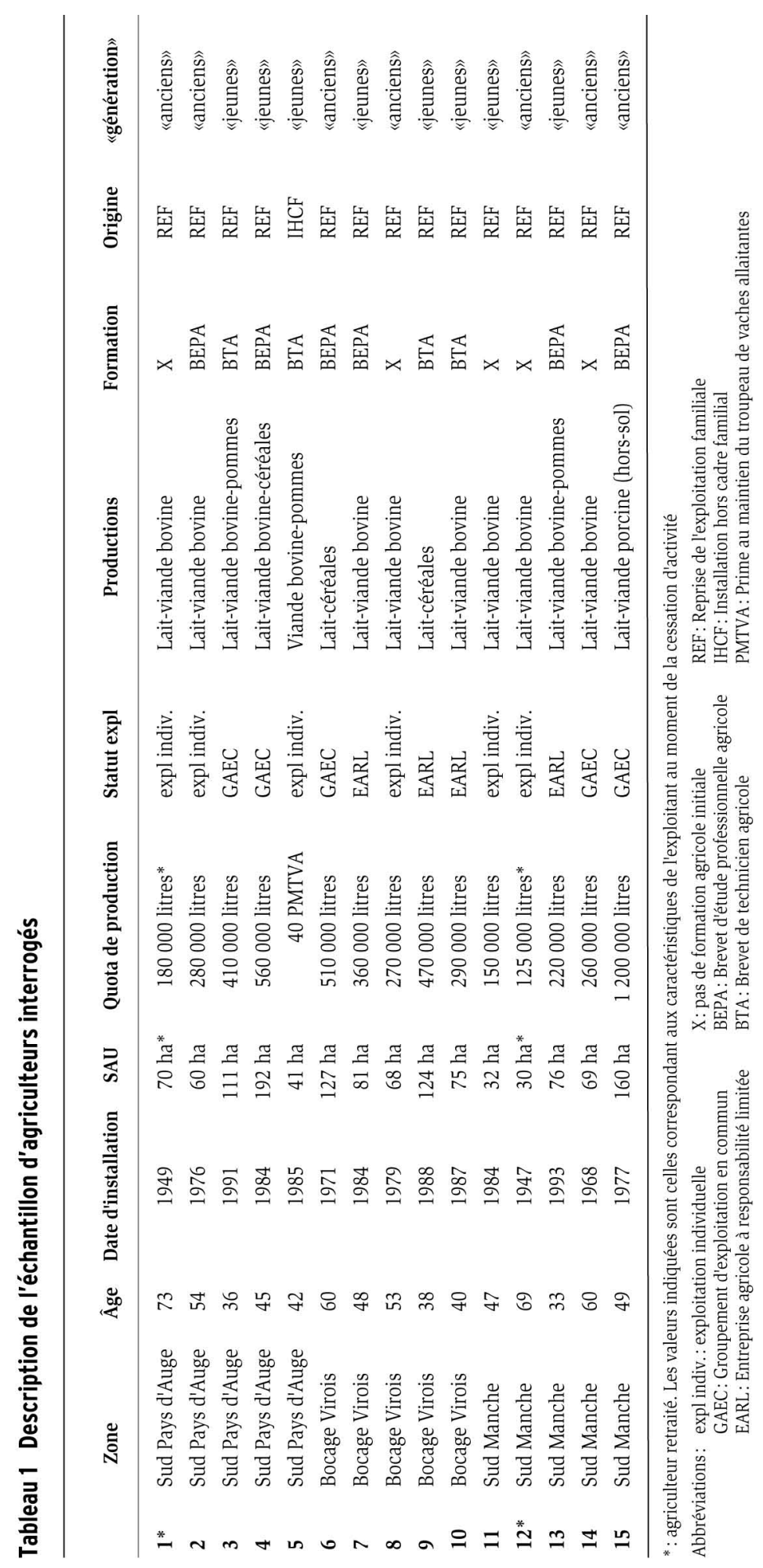


Les agriculteurs installés avant ou durant cette période ont presque tous pris une part active aux actions qui ont généré d'importantes transformations des systèmes de production et des paysages ruraux. Ils ont dû remettre en cause le système agraire de leurs parents pour construire une agriculture plus puissante et plus productive répondant aux demandes des consommateurs (Périchon, 2004). Pour y parvenir, il a fallu transformer radicalement les structures agraires des espaces de bocage et, par là même, remettre en cause certaines de leurs spécificités paysagères. Ces agriculteurs ont été les maillons de la transformation des paysages à l'échelle locale. Ce sont essentiellement eux qui ont abattu les haies, les pommiers et les poiriers, qui ont adopté la culture du maïs fourrage (Dionnet et al., 1973). Ce sont aussi eux qui ont construit de nouveaux bâtiments agricoles, délaissant ainsi le bâti agricole traditionnel devenu obsolète pour l'agriculture «moderne» (Madeline, 2006). Cette phase de leur activité professionnelle a durablement marqué leurs représentations paysagères et leurs opinions sur les évolutions passées des paysages de bocage (Le Caro, 2007 ; Javelle, 2007).

À partir de cette distinction générationnelle, la comparaison des clichés réalisés sur le thème de la définition de ce qu'est, selon les agriculteurs, une "belle parcelle» prend tout son sens (figure 3). Les agriculteurs les plus âgés ont généralement photographié des parcelles en culture (maïs ou céréales) de grande dimension, plates et peu bocagères ; c'est-à-dire des parcelles dont les caractéristiques correspondent aux besoins de l'agriculture intensive conventionnelle. Au contraire, les prises de vue des agriculteurs de la jeune génération montrent des parcelles généralement occupées par des prairies, assez bocagères et parfois même plantées de pommiers haute-tige. Ce type de parcelle fait explicitement référence à un modèle paysager qui par bien des aspects, peut apparaître comme opposé au précédent.

Plus généralement, deux grandes catégories de formes paysagères sont identifiées par les agriculteurs: d'une part les formes paysagères dites traditionnelles (haiesbocage, vergers haute-tige, prairies, bâti traditionnel) et, d'autre part, les formes paysagères issues directement ou indirectement de l'intensification des productions agricoles en cours depuis les années 1960-1970. La comparaison des clichés réalisés par les agriculteurs des deux groupes montre ainsi des différences majeures. Tous registres confondus, les représentations des jeunes agriculteurs semblent nettement plus marquées par les formes paysagères «traditionnelles» que celles des agriculteurs plus âgés (figure 3).

De prime abord, ce constat peut paraître paradoxal, mais lorsqu'on prend en compte le contexte de la patrimonialisation croissante des paysages ruraux en lien avec le développement des activités agrotouristiques, on comprend mieux les enjeux que recouvre la conservation de paysages ruraux originaux dans un contexte agricole relativement difficile. Le fait que les agriculteurs installés avant 1980 aient mentionné plus fréquemment les formes produites par l'intensification agricole est intéressant dans la mesure où ces formes paysagères ont été produites par les exploitants de cette même génération. Le faible nombre de références à ces formes explicitement formulées par les agriculteurs plus jeunes se traduit par un sentiment globalement critique vis-à-vis du système agricole productiviste. Il faut noter que cette jeune génération identifie bien les demandes émanant des sociétés urbaines et voit dans le développement agrotouristique l'occasion réelle de diversifier ses sources de revenu et de redynamiser 
les tissus économiques et sociaux des espaces ruraux. Cette attitude montre que les représentations paysagères des agriculteurs expriment des projets de développement pour les activités agricoles et, plus généralement, pour l'espace rural (figure 3).

\section{Figure 3 Différenciation générationnelle des représentations des agriculteurs}
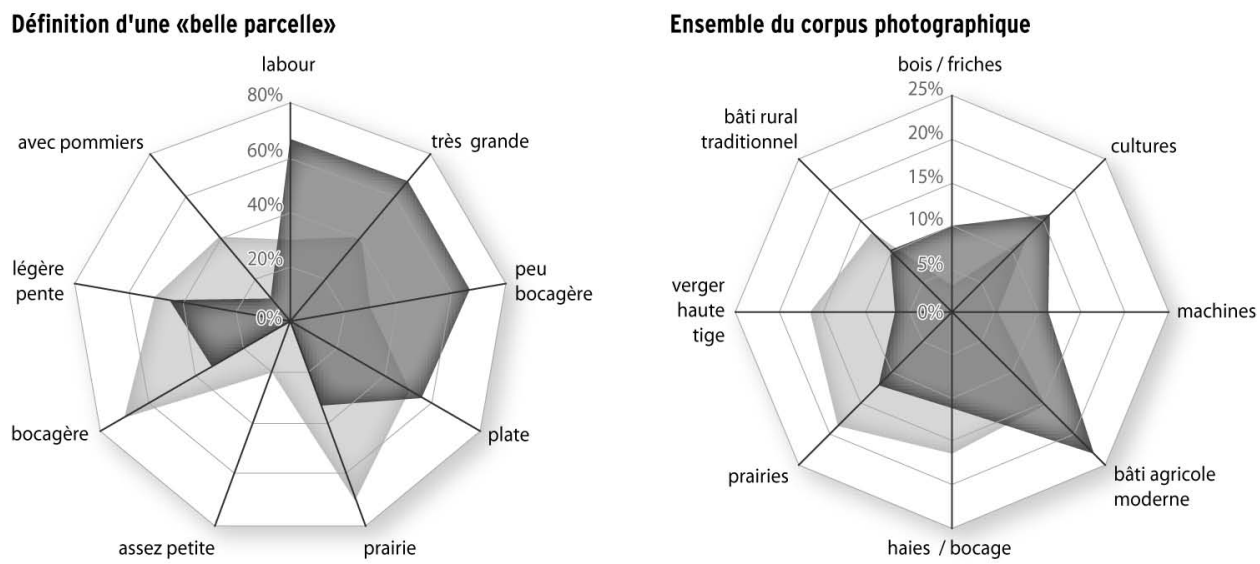

Anciens» (installés avant 1980)

«Jeunes agriculteurs» (installés après 1984)

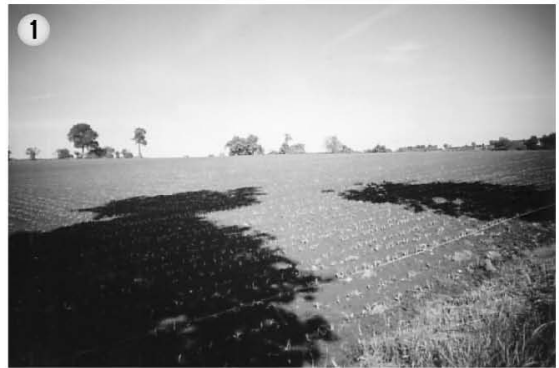

Vassy (14), Bocage Virois, mai 2008

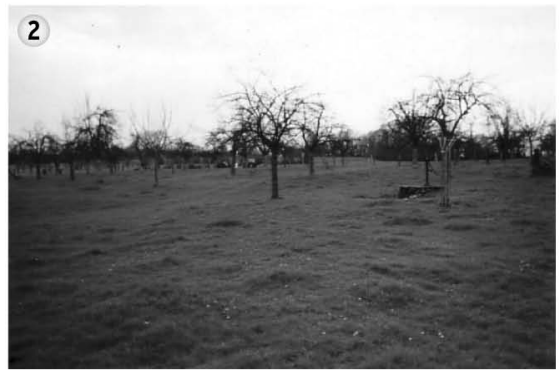

Le Renouard (61), Sud Pays d'Auge, avril 2004

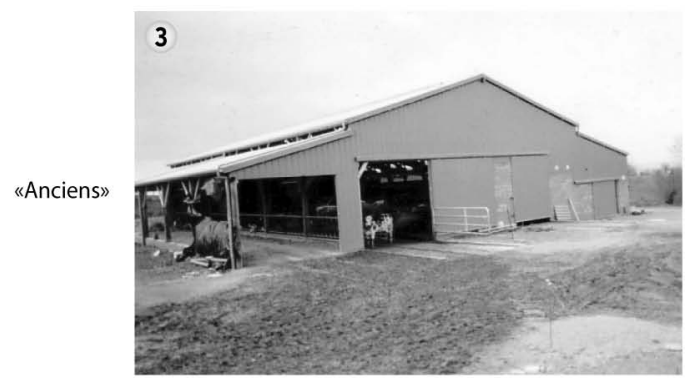

Le Teilleul (50), Sud Manche, avril 2004

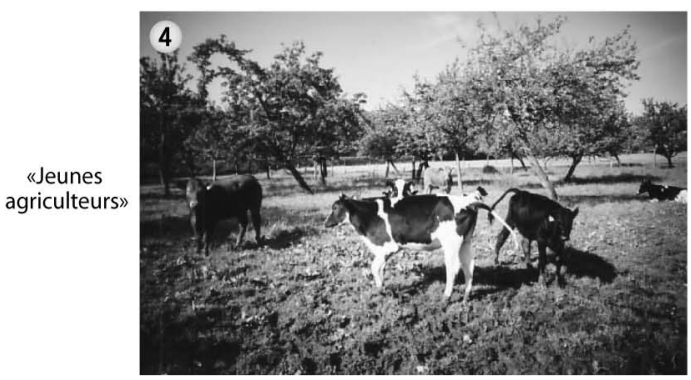

Vassy (14), Bocage Virois, juin 2008

Par ailleurs, leurs actions concrètes sur le paysage dans l'exercice de leur métier poussent fréquemment les exploitants à confronter leurs conceptions des espaces ruraux et de leur développement à celles qu'en ont d'autres types d'usagers 
(Le Caro, 2007). Certains agriculteurs agissent donc sur leurs pratiques agricoles et sur les paysages qui les entourent dans le cadre d'une valorisation ponctuelle des paysages ou de la régulation de conflits avec des populations non agricoles (surtout en ce qui concerne l'intégration paysagère des bâtiments d'élevage et l'arasement des haies ou des talus). Leur attitude apparaît comme pragmatique dans ce type de situation. Là encore, une étude plus approfondie de ces aspects conflictuels éclaire les décisions des exploitants dans un contexte de forte pression sociale. Le paysage recouvre ici une dimension symbolique dans l'expression des luttes de pouvoir à l'échelle locale (Sautter, 1985 ; Donadieu, 2002).

De nombreux agriculteurs font ainsi référence à la «pression» qu'ils ressentent lorsqu'ils transforment l'espace et agissent directement sur les structures paysagères (Le Caro, 2007). Ils mentionnent en effet fréquemment les conflits qui les opposent aux populations non agricoles ou les discussions et les débats que leur imposent ces populations lorsqu'elles constatent l'arasement d'une haie ou d'un talus, la mise en culture d'une parcelle en prairie (surtout les néoruraux et les résidants secondaires). Dans le Bocage Virois, Éric H. (38 ans), nous explique ainsi à ce propos :

Moi j'y vais doucement, enfin je veux dire pour ne pas me faire taper dessus au niveau du changement du paysage... j'y vais que par petits bouts... Bon, parce que d'abord au point de vue temps, ça demande du temps, et puis, ça choque moins les gens, c'est surtout ça... Il y aurait que moi, il y aurait bien moins de talus que ce qu'il y a aujourd'hui...

Ce témoignage est révélateur de la pression ressentie par les exploitants, sans nécessairement que ces derniers aient de contacts directs avec les populations non agricoles. Dans d'autres cas, ces contacts peuvent être directs et le conflit apparaît alors parfois comme ouvert entre l'agriculteur et ses voisins. Dans le Pays d’Auge, Régis L. (45 ans) évoque, par exemple, ses relations conflictuelles avec un résidant secondaire voisin de son exploitation:

\begin{abstract}
Et puis là, ce chemin là, au remembrement on a fait sauter la haie pour l'élargir, parce que bon il était pas carrossable, donc, bon, bah, on a arraché la haie... Et puis là, ça a été la catastrophe, parce que là, y a un parisien qu'est là, et il m’a incendié le jour où le bull est venu arracher la haie... [silence]... Bah, oui, forcément, parce que c'est moi qu'a demandé quand même à ce qu'on refasse ce chemin... Parce que avant, pour aller exploiter le bas de la ferme, on faisait un grand détour... Trois kilomètres aller, et trois kilomètres retour... Alors que c'est même pas à cinq cents mètres de la ferme. Donc la haie, on l'a arrachée pendant le remembrement... Bon, pour se rassurer, c'était qu'une haie d'ormes qu'étaient presque tous morts... Donc, il m’a incendié, le jour que le bull était là, il m’a dit : «oui, vous arrachez tout!», moi, je lui ai dit : «écoutez, vous vous venez deux fois par an, moi je suis à travailler ici tous les jours, et je me sens pas coupable de toucher au paysage »... Et puis encore aujourd'hui, quinze ans après, on se parle pas... Mais il sait bien aujourd'hui qu'il a fait une connerie, parce que tout ce qui a été arraché là a été replanté, et je peux te dire que c'est une belle haie maintenant.
\end{abstract}

Ce récit est assez révélateur des conflits qui opposent parfois les agriculteurs aux autres usagers de l'espace rural. Les premiers avouent ne pas toujours bien comprendre les manifestations de colère ou de désapprobation des néoruraux envers leurs techniques de production et les aménagements de leur territoire d'exploitation. Les agriculteurs interrogés semblent toutefois assez favorables aux mesures de gestion «raisonnée» des paysages instaurées par l'État ou les collectivités locales. Mais ce sentiment varie selon la génération à laquelle ils appartiennent. 
En général, les exploitants les plus âgés semblent moins sensibles aux arguments d'une gestion paysagère permettant le développement de l'agrotourisme. Ils semblent plus ouverts à une gestion paysagère et environnementale peu contraignante pour les activités agricoles. Au contraire, les agriculteurs les plus jeunes présentent une plus grande sensibilité aux possibilités offertes par l'agrotourisme, comme alternative, ou du moins comme complément, aux activités agricoles classiques. Ils acceptent donc mieux les contraintes productives liées à la préservation et à la gestion des formes paysagères "traditionnelles». D'ailleurs, ces jeunes agriculteurs acceptent plutôt bien la mise en place de certaines aides ou indemnités compensatoires, comme des contrats territoriaux d'exploitation (CTE) ${ }^{2}$, qui ont permis à certains d'entre eux de replanter des pommiers haute-tige et des haies, ou d'implanter des dérobés de raygrass pendant la période d'interculture.

Pour une partie des agriculteurs, le CTE fut bien sûr l'occasion de mettre en place des pratiques innovantes localement, plus respectueuses de l'environnement et permettant de remettre en valeur certaines zones délaissées de l'espace agricole (pâture des coteaux calcaires enfrichés dans le Pays d'Auge, par exemple). La pratique du dérobé dans le Bocage Virois faisait par exemple partie d'un «CTE type» dans cette région. Alain L. (48 ans) exprime ainsi ses motivations pour la mise en place de dérobés de ray-grass:

\begin{abstract}
En fait, je l'ai fait dans le cadre d'un CTE, j'en faisais déjà un petit peu avant, donc j'ai augmenté les surfaces dans le cadre du CTE; bon mon CTE se termine, mais je crois que je vais continuer quand même. Pour moi, ça a un double avantage parce que j'en retire un profit derrière aussi, et puis quand on voit la pousse de ray-grass qu'on a, même très tôt suivant le temps qu'il fait au printemps, ou même des fois à l'automne aussi, on se dit que, vu la couleur, on a sûrement récupéré quelques points d'azote... C'est toujours tant mieux plutôt que de les laisser filer vers les rivières et les sources. Parce que quand on voit un ray-grass au mois de septembre ou octobre qu'est bien vert, on se dit qu'y avait ce qu'y fallait dans le sol.
\end{abstract}

Si la motivation d'Alain L. est directement liée à un souci de préservation de l'environnement, la dimension économique reste un argument d'importance. À cet exploitant, le CTE a permis de mettre en place la pratique du dérobé à moindre coût et d'en pérenniser l'usage.

Parmi les enseignements de ce travail de terrain, nous pouvons affirmer que les jeunes agriculteurs paraissent ainsi plus sensibles à la préservation de l'environnement que leurs aînés. Cette sensibilité environnementale se combine à leur sensibilité paysagère pour la conservation de certaines formes paysagères dont le rôle environnemental est intégré. La haie semble être la meilleure illustration de ce phénomène étant donné, d'une part, son caractère structurant et emblématique qui en fait un élément fort des paysages de bocage et, d'autre part, ses divers rôles environnementaux (abri pour la faune, brise-vent, régulateur de l'érosion et du ruissellement...). Cette sensibilité conduit les agriculteurs à mieux accepter les actions de protection et de gestion des réseaux de haies, qu'ils considèrent alors comme légitimes (Rémy, 2003).

2 Les CTE ont été mis en place en 1999 puis remplacés en 2002 par les Contrats agriculture durable (CAD); on retrouve aujourd'hui des mesures similaires sous le nom de Mesures agro-environnementales territorialisées (MAET). 
Enfin, pour essayer de dépasser ce clivage générationnel et revenir à un niveau plus collectif, le discours porté par les agriculteurs à l'égard du paysage renvoie souvent à des références et à des valeurs largement partagées au sein du monde agricole (Dupont, 1996). Ces références font directement appel à ce que Prével (2007) nomme «l’imaginaire du productivisme agricole» dans lequel les notions de progrès, de performance et de maîtrise technique sont omniprésentes. C'est donc bien à la construction des identités professionnelles que peut être associé ce travail sur les rapports qu'entretiennent les agriculteurs avec les paysages.

\section{La maîtrise technique et le contrôle de la nature dans le discours des agriculteurs}

L'imaginaire du productivisme agricole, et notamment la place qu'y occupent la technique et la nature, se traduit directement dans la manière dont les agriculteurs conçoivent leur métier, sur la manière dont ils se définissent en tant que groupe professionnel et sur la place qu'ils doivent occuper dans la société française (Hervieu et Viard, 2001; Darré, 2004; Rémy, 2005). Ainsi, la prise en compte des différents systèmes de valeurs existants au sein du monde agricole permet de comprendre l'importance des modèles de références et de normes de comportement dans les pratiques des agriculteurs (Peyre, 2004 ; Caillault et Marie, 2009).

Prenons le cas des friches (figure 4, photos nos 1,2 et 3 ). Pour un grand nombre d'exploitants, elles cristallisent le rejet et les sentiments négatifs à l'égard du paysage. Elles sont ainsi souvent associées au recul des activités agricoles dans l'occupation de l'espace et à la baisse du nombre d'agriculteurs (Luginbühl, 1993; Soulard et Pierret, 1996). À l'examen de la photo no 1 (figure 4), un agriculteur (38 ans) apporte le commentaire suivant : «Les endroits comme ça... ça fait peine à voir, on se dit que c'est triste... y a plus de vie...».

Dans les propos des agriculteurs, l'évocation de la friche conduit à un discours récurrent sur le travail. L'exemple d'un agriculteur du Pays d'Auge (54 ans), auteur d'un cliché mettant en scène une parcelle en cours d'enfrichement, est révélateur : «Souvent sur les buttes ça s'encrasse... Alors, bon, si le gars il est courageux, ça va, y nettoie, mais... si il sait pas bosser, ça reste comme ça» (figure 4, photo no 2 ).

Un discours qu'on retrouve chez un autre exploitant du Sud Manche (47 ans) : «Si on va dans une région où c'est en friche, c'est que les agriculteurs ne travaillent pas de trop... Par exemple, ma femme, elle a une partie de sa famille dans le coin d'Orbec... Et bah, je peux vous dire que là-bas, les haies elles sont mal entretenues, les gars y doivent pas être débordés par le travail là-bas, ça, c'est sûr».

Ces propos renvoient à la place des activités agricoles dans les espaces ruraux, surtout en Basse-Normandie où la surface agricole utile occupe souvent la majeure partie du territoire. Une part très significative des agriculteurs normands, imprégnés par l'idéologie du progrès et de la modernisation agricole, refusent en effet toute idée de déprise ou de recul de l'emprise de l'agriculture dans l'espace. Pour eux, les friches sont des éléments symptomatiques de la diminution du nombre d'exploitations et de la part des agriculteurs dans les sociétés rurales (Périchon, 2005). D’autres, plus rares et installés suivant une trajectoire atypique (souvent extérieure au milieu agricole), 
considèrent en revanche la friche comme un élément du paysage à part entière qui, à leur yeux, n’apparaît pas nécessairement comme quelque chose de négatif. Dans le Pays d'Auge, Mlle F. (42 ans) s'exprime par exemple en ces termes : «les friches, elles me gênent pas tant que ça, ça fait un paysage... plus riche en arbres».

\section{Figure 4 Les friches et les cultures dans les clichés des agriculteurs}

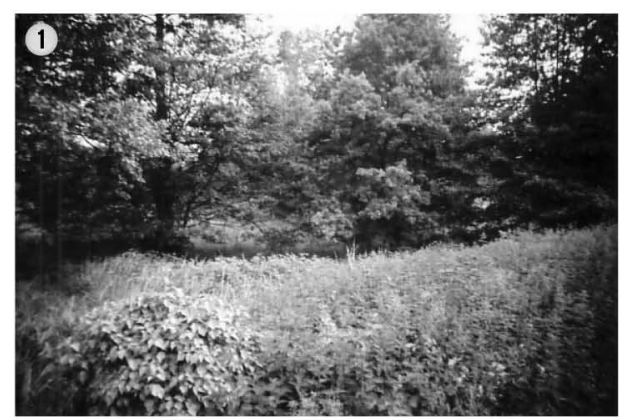

Vassy (14), Bocage Virois, juin 2008

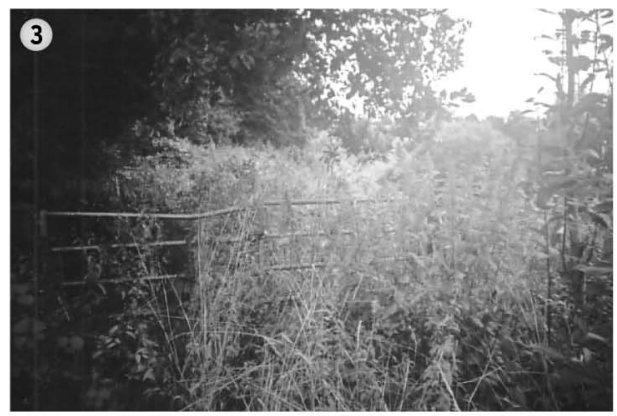

Rully (14), Bocage Virois, juin 2008

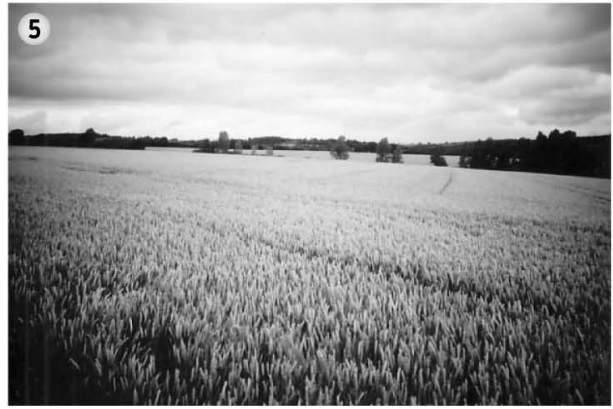

Lassy (14), Bocage Virois, juin 2008

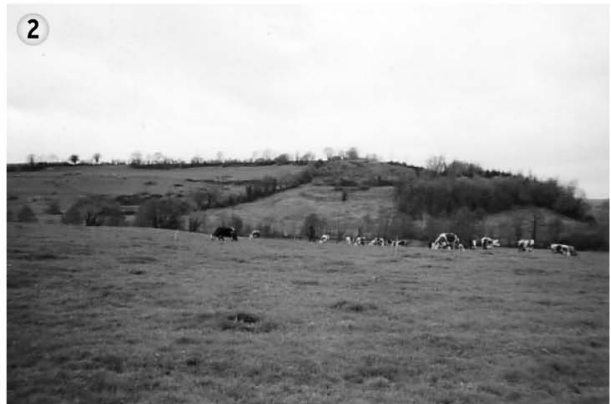

Castillon-en-Auge (14), Pays d'Auge, avril 2004
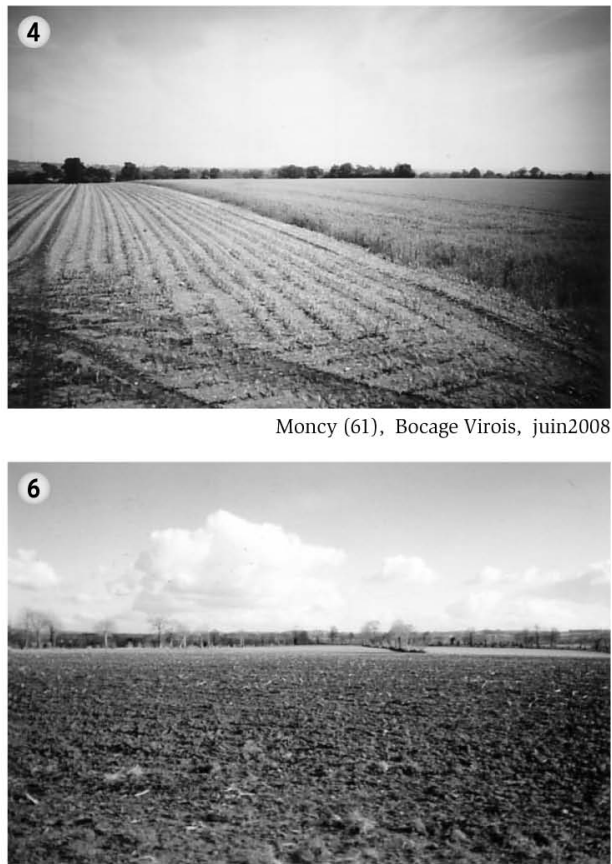

Sainte-Marie-du-Bois (50), Sud Manche, avril 2004

Au contraire, lorsque les exploitants agricoles évoquent les éléments associés à la culture du maïs fourrage ou des céréales (grandes parcelles aux formes simples, figure 4 : photos nos 4,5 et 6 ), les discours recouvrent une dimension beaucoup plus positive (Guisepelli, 2005). La parole d'un agriculteur du Sud Manche (60 ans) en offre la démonstration: «Là au moins, c'est propre, c'est net. Et puis, y a pas de pointe et y a de la place... enfin, j'veux dire, c'est pas trop petit... c'est plus moderne quoi... pour le travail» (figure 4 : photo $n^{\circ} 6$ ). 
Un autre (40 ans), dans le Bocage Virois, confie que: «[les cultures] ça donne une image dynamique des exploitations, parce que bon quand on voit des cultures bien menées, on se dit que les agriculteurs y sont au point... niveau technique et niveau boulot» (figure 4 : photo $n^{\circ} 5$ ).

Les cultures et le labour sont aussi une source de plaisir et de satisfaction pour les agriculteurs. Certains d'entre eux les évoquent dans des termes qui expriment sans ambiguïté la dimension esthétique du «travail bien fait». Dans le Bocage Virois, par exemple, un exploitant (38 ans) commente un de ses clichés: «Une parcelle comme ça... c'est beau... [silence]... Pour le gars qu'a déjà pris une charrue, ça laisse rêveur...» (figure 4: photo $\mathrm{n}^{\mathrm{0}} 4$ ).

En effet, dans l'esprit d'une grande partie des agriculteurs interrogés, l'utilisation de la machine est fortement valorisée, car elle fait appel à leur imaginaire et aux notions de maîtrise technique et de capacité de travail. Les machines occupent donc une place de choix sur les photographies. Les rapports qu'entretiennent les exploitants avec les engins agricoles et les divers équipements utilisés n’apparaissent que rarement comme de simples rapports utilitaires (Saugeres, 2002). Ils sont en effet marqués par une forte dimension symbolique issue de l'idéologie du progrès et de la modernisation agricole (Prével, 2007). Les prises de vue où les machines sont présentes révèlent souvent une volonté de mise en scène des engins ou de choix de ceux qui "méritent» d'y figurer (le tracteur le plus moderne, le plus gros, l'importance du parc motorisé, l'ensileuse). Le type d'engins représentés fait ainsi très souvent appel à l'idée de puissance et de maîtrise technique et agronomique de la production. Les machines utilisées pour la conduite des opérations techniques relatives aux cultures de maïs et de céréales semblent ainsi particulièrement mises en avant: tracteurs puissants, pulvérisateurs, ensileuses et charrues (figure 5).

Cependant, la friche est perçue par tous comme le révélateur des transformations du métier: elle résulte de l'augmentation de la productivité, mais aussi de la charge de travail. Celui qui n’arrive plus à faire face à cette charge de travail est alors stigmatisé comme un «fainéant». Le rapport au travail, la capacité de travailler et de produire toujours davantage, est au cœur de la perception qu'ont les agriculteurs de la friche (Prével, 2007). Pour eux, les éléments du paysage portent donc différentes valeurs symboliques liées aux représentations qu’ils ont de leur propre métier.

Les équipements liés à la récolte de l'herbe (un cliché de faucheuse, aucun de faneuse, aucun de presse à grosse balle) et ceux liés aux animaux (deux clichés de salle de traite, aucun de dessileuse ou de pailleuse, un de mélangeuse) sont au contraire presque absents des photographies, alors qu'ils sont probablement les plus fréquemmement utilisés par les agriculteurs. Cet élément révèle bien la valorisation dont sont l'objet les opérations techniques les plus mécanisées et celles faisant appel aux engins les plus puissants, mais il renvoie aussi aux valeurs très positives associées aux cultures (maïs et céréales) dans les représentations des agriculteurs. 


\section{Figure 5 Exemples de clichés d'engins agricoles réalisés par les agriculteurs}

Des engins qui symbolisent le progrès et la maîtrise technique...

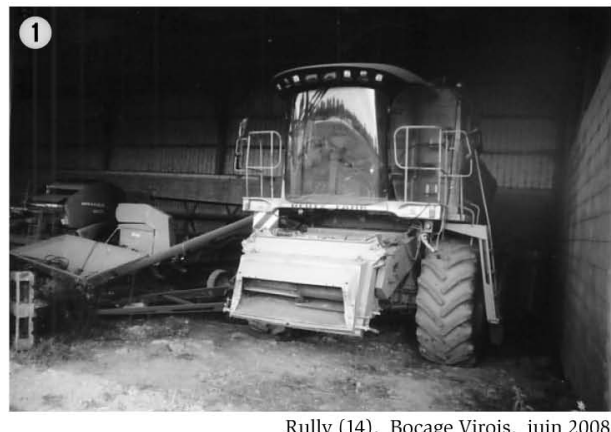

Rully (14), Bocage Virois, juin 2008

La mise en scène de la machine...

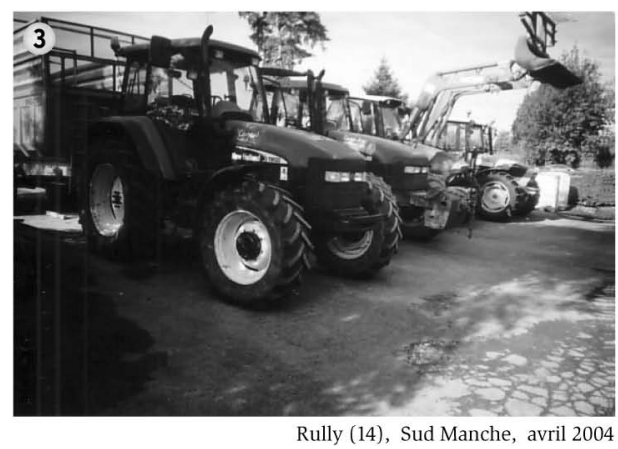

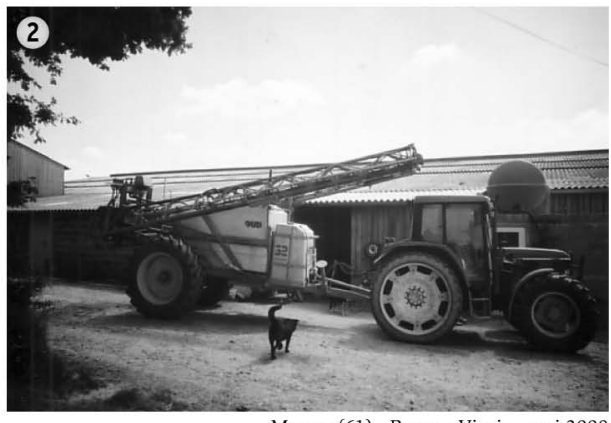

Moncy (61), Bocage Virois, mai 2008

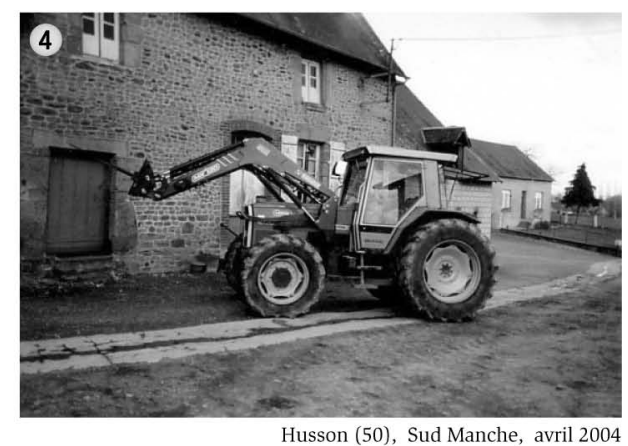

\section{Conclusion}

Les résultats de cette enquête confirment que l'étude des représentations paysagères des agriculteurs est essentielle pour analyser leurs pratiques. La méthode retenue pour conduire cette étude s'appuie sur la distribution d'appareils photo jetables ainsi que sur l'analyse des clichés et des discours produits par les exploitants agricoles bas-normands (Michelin, 1998; Marie, 2007). Cette méthode, combinée au travail d'entretien, permet d'obtenir des résultats intéressants quant aux rapports des agriculteurs au paysage. Le caractère participatif de la démarche permet également de donner aux individus une grande liberté dans les choix de prise de vue, et de limiter ainsi les effets d'imposition et de subjectivité apportés par le chercheur. L’intérêt de la méthode réside ici dans la production d'un matériau très riche pour le chercheur, mais sa principale limite demeure le temps et l'investissement nécessaire au travail d'enquête, rendant sa systématisation difficile.

L'étude des représentations paysagères des agriculteurs met d'abord en lumière leur grande sensibilité à l'égard du paysage et de ses transformations récentes (Ambroise et al., 2000; Marie, 2007). Leur lecture du paysage et de ses évolutions est par ailleurs essentiellement structurée autour des formes produites par les activités agricoles (Cadiou et Luginbühl, 1995 ; Le Caro, 2007). La dimension symbolique que recouvrent les liens entre ces activités et le paysage apparaît aussi centrale pour comprendre de manière globale les représentations des agriculteurs, comme l'illustre l'exemple de l'enfrichement et de la déprise agricole (Cadiou, 1991 ; Soulard et Pierret, 1996). 
Par ailleurs, le facteur générationnel intervient en première instance dans de nombreux cas. En effet, la participation à l'intensification des systèmes de production paraît influencer les agriculteurs les plus âgés dans leurs représentations des formes paysagères «traditionnelles », qui y sont très peu valorisées. Au contraire, les représentations des exploitants installés après les graves crises de surproduction des années 1980 - correspondant aux premières remises en cause, dans le monde agricole, du modèle productiviste - semblent beaucoup plus fortement marquées par le rejet des formes paysagères issues de l'intensification agricole et valorisent davantage les formes «traditionnelles». Ce clivage générationnel révèle en fait les profondes recompositions des identités professionnelles en cours au sein du monde agricole depuis la fin des années 1960.

Les modèles paysagers auxquels se réfèrent les individus sont donc très différents selon les conceptions de l'activité agricole et de ses missions dans les espaces ruraux (Marie, 2007). À travers les clichés de «belles parcelles», l'étude montre des oppositions très nettes en fonction du positionnement des agriculteurs par rapport au modèle d'exploitation de référence défini suivant les normes et les valeurs du productivisme agricole (Darré, 2004 ; Marie, 2009). L'analyse des discours sur les friches et les cultures révèle également l'importance des identités professionnelles dans la construction des représentations paysagères (Périchon, 2003). Les rapports au travail, à la nature et à la technique apparaissent donc comme des leviers essentiels pour la compréhension des relations entretenues par les agriculteurs avec le paysage. La diversité des représentations paysagères renvoie enfin aux clivages politiques et idéologiques qui traversent le monde agricole et au changement social qui a accompagné la modernisation agricole (Lémery, 2003 ; Bessière, 2004; Rémy, 2006). Elle traduit ainsi des conceptions très différentes de l'activité agricole et de sa place dans l'entretien des paysages et de l'environnement dans les espaces ruraux et, plus largement, de la place de l'agriculture dans la société (Miéville-Ott et Droz, 2010). 


\section{Bibliographie}

AMBROISE, Régis, BONNEAUD, François et BRUNET-VINCK, Véronique (2000) Agriculteurs et paysages. Dix exemples de projets de paysage en agriculture. Dijon, Éditions Éducagri.

BECKER, Howard (2007) Les photographies disent-elles la vérité? Ethnologie française, $n^{\circ} 1$, p. 33-42.

BERMOND, Michaël (2004) Produire et se reproduire dans la crise. Agriculture, familles, exploitations en Normandie au début du XXIe siècle. Essai de géographie sociale. Université de Caen. Thèse de doctorat.

BERTRAND, Magali et BORIES, Olivier (2004) Distinctions paysagères et distinctions sociales pour questionner les individus sur leur rapports aux territoires ruraux et périurbains. Colloque «Faire campagne», Université de Rennes 2. [En ligne.] http:// eso.cnrs.fr/spip.php?article370

BESSIÈRE, Céline (2004) Vaut mieux qu'elle travaille à l'extérieur! Enjeux du travail salarié des femmes d'agriculteurs dans les exploitations familiales. Cahiers du genre, n³7, p. 93-114.

CADIOU, Nathalie (1991) Perceptions du paysage dans le Domfrontais et l'évolution de l'espace rural. Études rurales, nos 121-124, p. 127-139.

CADIOU, Nathalie et LUGINBÜHL, Yves (1995) Modèles paysagers et représentations du paysage en Normandie-Maine. Dans Claudie Voisenat (1995) Paysage au pluriel: pour une approche ethnologique des paysages. Paris, Maison des sciences de l'homme.

CAILlAULT, Sébastien et MARIE, Maxime (2009) Pratiques agricoles, perceptions et représentations du paysage: quelles articulations? Approches croisées Nord/ Sud. Norois, vol. 213, nº 4, p. 9-20.
CALVO-IGLESIAS Silvia, CRECENTEMASEDA, Rafael et FRA-PALEO, Urbano (2006) Exploring farmer's knowledge as a source of information on past and present cultural landscapes. A case study from NW Spain. Landscape and urban planning, vol. 78, nº 4, p. 334-343.

CHOSSON, Jean-François (2003) Les générations rurales, 1945-2002. Paris, LGDJ.

COLLIER, John Jr et COLLIER, Malcolm [1967] (1986) Visual anthropology: Photography as a research method. Albuquerque, University of New Mexico Press.

CONORD, Sylvaine (2007) Usages et fonctions de la photographie. Ethnologie française, vol. 1, nº37, p. 11-22.

DARRÉ, Jean-Pierre, MATHIEU, Anne et LASSEUR, Jacques (2004) Le sens des pratiques. Conceptions d'agriculteurs et modèles d'agronomes. Paris, INRA-Science Update.

DIONNET, Marie-Claire, HERIN, R. et PRUDHOMMEAUX, S. (1973) Le maïs en BasseNormandie: facteurs économiques d'un succès et limites prévisibles d'une réussite. Cahiers du département de géographie de l'Université de Caen, nº 7, p. 19-42.

DONADIEU, Pierre (2002) La société paysagiste. Arles-Versailles, Éditions Actes-Sud/ ENSP.

DUPONT, Yves (1996) La passion du déracinement et de l'innovation ou la science et la technique comme idéologie. Enquêtes rurales, $\mathrm{n}^{\circ} 1$, p. 83-101.

DROZ, Yvan, MIÉVILLE-OTT, Valérie, FORNEY, Jérémie et SPICHIGER, Rachel (2009) Anthropologie politique du paysage. Valeurs et postures paysagères des montagnes suisses. Paris, Éditions Karthala.

GUISEPELLI, Emmanuel (2005) Les représentations sociales du paysage comme outils de connaissance préalable à l'action. L'exemple des Alpes du nord. Cybergéo. [En ligne.] http://cybergeo.revues. org/3352 
GRAVSHOLT BUSCK, Anne (2002) Farmers' landscape decisions: Relationships between farmers' values and landscape practices. Sociologia Ruralis, vol. 42, n ${ }^{\circ} 3$, p. 233-249.

HARPER, Douglas (2002) Talking about pictures: A case for photo elicitation. Visual Studies, vol. 1, no 17, p. 13-26.

HERVIEU, Bertrand et VIARD, Jean (2001) L'archipel paysan. La fin de la république agricole. La Tour d'Aigues, Éditions de l'Aube.

JAVELLE, Aurélie (2007) Perceptions de la biodiversité par des agriculteurs sur une zone atelier du nord-est de la Bretagne et évaluation de leur rencontre avec des chercheurs en environnement ou La main et le stylo. Université de Rennes I, thèse de doctorat. [En ligne.] http://tel.archivesouvertes.fr/docs/00/19/59/67/PDF/ thesemise_en_forme.pdf

LE CARO, Yvon (2007) Les loisirs en espace agricole. L'expérience d'un espace partagé. Rennes, Presses Universitaires de Rennes.

LELLI, Laurent et PARADIS, Sylvie (2005) Analyse critique d'un dispositif méthodologique de diagnostic paysager: le cas du bassin versant du Cérou (Tarn, Midi-Pyrénées). Géocarrefour, vol. 80, n²2, p. 123-130.

LÉMERY, Bruno (2003) Les agriculteurs dans la fabrique d'une nouvelle agriculture. Sociologie du travail, vol. 45, n ${ }^{\circ}$, p. 9-25.

LUGINBÜHL, Yves (1991) Le paysage rural. La couleur de l'agricole, la saveur de l'agricole, mais que reste-t-il de l'agricole? Études rurales, nos 121-124, p. 27-44.

LUGINBÜHL, Yves (1993) Sauvage - cultivé: l'ordre social de l'harmonie des paysages. Dans Nicole Mathieu et Marcel Jollivet (dir.) (1993) Du rural à l'environnement. La question de la nature aujourd'hui. Paris, ARF éditions - L'Harmattan.
LUGINBÜHL, Yves (1995) Quelques avatars de la recherche sur le paysage. Dans Claudie Voisenat (1995) Paysage au pluriel: pour une approche ethnologique des paysages. Paris, Éditions de la Maison des sciences de l'homme.

MADELINE, Philippe (2006) Les constructions agricoles dans les campagnes françaises. Héritages et dynamiques actuelles d'évolution. Histoire et Sociétés Rurales, no26, p. 53-93.

MARESCA, Sylvain (2007) Photographes et ethnologues. Ethnologie française, $\mathrm{n}^{\circ} 1$, p. 61-67.

MARIE, Maxime (2007) Deux générations d'agriculteurs face aux transformations des paysages bocagers. Étude de cas en Normandie. Cahiers d'économie et de sociologie rurales, no84-85, p. 192-214.

MARIE, Maxime (2009) Des pratiques des agriculteurs à la production de paysage de bocage. Étude comparée des dynamiques et des logiques d'organisation spatiale des systèmes agricoles laitiers en Europe (Basse-Normandie, Galice, Sud de l'Angleterre). Université de Caen Basse-Normandie, Thèse de doctorat. [En ligne.] http://tel.archives-ouvertes.fr/ docs/00/44/11/17/PDF/These_MaximeMARIE_2009.pdf

MÉNADIER, Lydie (2010) Que révèlent «parcelles préférées» et "coins de paradis" sur les caractères d'un produit? Méthode d'analyse du point de vue paysager d'agriculteurs en zones AOC fromagères de moyenne montagne. Projets de paysage - Revue scientifique sur la conception et l'aménagement de l'espace, no 4 . [En ligne.] http://www. projetsdepaysage.fr/fr/que_revelent_parcelles_preferees_et_coins_de_paradis_ sur_les_caracteres_d_un_produit_

MICHELIN, Yves (1995) Les jardins de Vulcain: paysages d'hier, d'aujourd'hui et de demain dans la chaîne des Puys du massif central. Paris, Maison des sciences de l'Homme. 
MICHELIN, Yves (1998) Des appareils photo jetables au service d'un projet de développement: représentations paysagères et stratégies des acteurs locaux de la montagne thiernoise. Cybergéo. [En ligne.] http://cybergeo.revues.org/5351

MIÉVILLE-OTT, Valérie et DROZ, Yvan (2010) Évolution de la réflexion paysagère en Suisse. À partir du programme Paysages et habitats de l'arc alpin. Économie rurale, no315, p. 46-59.

MOORE, Susan (1997) "Place" and sustainability: research opportunities and dilemmas. Dans Frank Vanclay et Luciano Mesiti (dir.) Sustainability and social research. Proceedings of the conference of the Australian Association for social research. Centre for Rural Social Research Charles Sturt University.

PAPINOT, Christian (2007) Le malentendu productif. Réflexions sur la photographie comme support d'entretien. Ethnologie française, vol. 1, no37, p. 79-86.

PÉRICHON, Samuel (2003) L'impossible reconstruction des bocages détruits. Quand l'évolution des représentations sociales associées au bocage explique l'échec des politiques de replantation de haies dans les communes du Sud-Est de l'Ille-et-Vilaine. L'espace géographique, vol. 2, no33, p. 175-187.

PÉRICHON, Samuel (2004) Les paysages campagnards sont-ils à l'image de ce que les agriculteurs souhaitent montrer d'euxmêmes. Note de recherches de l'Académie d'Agriculture de France [En ligne.] http://www.academie-agriculture.fr/ mediatheque/_files/publications/notes_ recherche/200506note1.pdf

PÉRICHON, Samuel (2005) Les représentations sociales associées au bocage. Analyse des relations agriculteurs/territoires en Haute-Bretagne. Bulletin de la Société géographique de Liège, nº46, p. 71-80.
PEYRE, Dominique (2004) La recherche, le développement, et les canards boiteux: quatre éleveurs face au même modèle de référence. Dans Jean-Pierre Darré, Anne Mathieu et Jacques Lasseur (dir.) Le sens des pratiques. Conceptions d'agriculteurs et modèles d'agronomes. Paris, INRAScience Update.

PRÉVEL, Maxime (2007) L'usine à la campagne. Une ethnographie du productivisme agricole. L'Harmattan, Paris.

RÉMY, Jacques (2003) Agriculteurs, société, environnement. APCA-INRA, Paris. [En ligne.] http://www.ivry.inra.fr/mona/ publications_chercheurs/Textes-Publis/ Remy_A-S-E.pdf

RÉMY, Jacques (2005) Le paysage: culture et jardinage. Dans Yvan Droz et Valérie Miéville-Ott (dir.) La polyphonie du paysage. Lausanne, Presses polytechniques et universitaires romandes.

RÉMY, Jacques (2006) Une illusion bien fondée? Le groupe des agriculteurs. Les Mondes agricoles en politique, séminaire de recherche interdisciplinaire, CEVIPOFINRA-CIHEAM, séance du 16 octobre. [En ligne.] http://www.cevipof.msh-paris. $\mathrm{fr} /$ rencontres/smnr/mondes_agricoles/ V1/S1/V1S1200610_Txt_JRemy.pdf

SAUGERES, Lise (2002) Of tractors and men: Masculinity, technology and power in a french farming community. Sociologia Ruralis, vol. 42, n² , p. 143-159.

SAUTTER, Gilles (1985) Paysagismes. Dans Chantal Blanc-Pamard (dir.) À travers champs, agronomes et géographes. Paris, Éditions ORSTOM.

SOUDIÈRE (de la), Martin (1984) Cueillir la montagne. Les agriculteurs et la nature en Margeride: de l'usage des mots aux usages des lieux. Université de Paris 7/EHESS. Thèse de doctorat.

SOULARD, Christophe et PIERRET, Pascal (1996) Le rapport des agriculteurs à la friche: enquête dans l'Auxois (Côte d'Or). Dans Collectif (dir.) Espaces et nature dans la géographie d'aujourd'hui. Géopoint 96, Université d'Avignon. 